PROCEEDINGS OF THE

AMERICAN MATHEMATICAL SOCIETY

Volume 125, Number 9, September 1997, Pages 2681-2692

S 0002-9939(97)03960-9

\title{
A CHARACTERIZATION OF SEMIBOUNDED SELFADJOINT OPERATORS
}

\author{
SEPPO HASSI, MICHAEL KALTENBÄCK, AND HENK DE SNOO
}

(Communicated by Palle E. T. Jorgensen)

\begin{abstract}
For a class of closed symmetric operators $S$ with defect numbers $(1,1)$ it is possible to define a generalization of the Friedrichs extension, which coincides with the usual Friedrichs extension when $S$ is semibounded. In this paper we provide an operator-theoretic interpretation of this class of symmetric operators. Moreover, we prove that a selfadjoint operator $A$ is semibounded if and only if each one-dimensional restriction of $A$ has a generalized Friedrichs extension.
\end{abstract}

\section{INTROdUCTION}

Let $A$ be a selfadjoint relation in a Hilbert space $\mathfrak{H}$ with inner product [., .]. The one-dimensional symmetric restrictions of $A$ are in one-to-one correspondence with all one-dimensional subspaces $\operatorname{span}\{\varphi\} \subset \mathfrak{H}$, via

$$
S=\{\{f, g\} \in A:[g-\bar{\mu} f, \varphi]=0\},
$$

where $\mu \in \mathbb{C} \backslash \mathbb{R}$ is fixed. If $A$ is an operator and $E(t)$ is its spectral function, then

$$
f \in \operatorname{dom} S \text { if and only if } f \in \operatorname{dom} A \text { and } \int_{\mathbb{R}}(t-\bar{\mu}) d([E(t) f, \varphi])=0 .
$$

If $A$ is a semibounded selfadjoint relation, then each symmetric restriction $S$ is semibounded and

$$
S_{F}=\left\{\{f, g\} \in S^{*}: f \in \mathfrak{H}_{S}\right\}
$$

is a selfadjoint extension of $S$, called the Friedrichs extension. Here the Hilbert space $\mathfrak{H}_{S}$ is the completion of $\operatorname{dom} S$ with the inner product $[(S+a) f, g], f, g \in$ $\operatorname{dom} S$, where $a \in \mathbb{R}$ is any number such that $S+a$ has a positive lower bound. The extension $S_{F}$ is the unique selfadjoint extension $H$ of $S$ with the property $\operatorname{dom} H \subset \mathfrak{H}_{S}$, see e.g. [1] and [6].

We now introduce a generalization of the Friedrichs extension for symmetric operators, which are not necessarily semibounded. Let $S$ be a closed symmetric

Received by the editors April 22, 1996.

1991 Mathematics Subject Classification. Primary 47B15, 47B25.

Key words and phrases. Symmetric operator, selfadjoint extension, Friedrichs extension, $Q-$ function, Nevanlinna function.

The second author was supported by "Fonds zur Förderung der wissenschaftlichen Forschung" of Austria, Project P 09832-MAT.

(C) 1997 American Mathematical Society 
operator with defect numbers $(1,1)$ and let $A$ be a selfadjoint extension of $S$. Let $\mathfrak{H}_{+1, A}$ be the Hilbert space $\operatorname{dom}\left|A_{s}\right|^{\frac{1}{2}}$ provided with the inner product

$$
[f, g]+\left[\left|A_{s}\right|^{\frac{1}{2}} f,\left|A_{s}\right|^{\frac{1}{2}} g\right], \quad f, g \in \operatorname{dom}\left|A_{s}\right|^{\frac{1}{2}},
$$

where $A_{s}$ denotes the operator part of $A$. Let $\mathfrak{H}_{S, A}$ be the closure of $\operatorname{dom} S$ in $\mathfrak{H}_{+1, A}$. Then

$$
A_{S}=\left\{\{f, g\} \in S^{*}: f \in \mathfrak{H}_{S, A}\right\}
$$

defines a selfadjoint extension of $S$. In fact, $A_{S}$ is the only selfadjoint extension $H$ of $S$ with the property that $\operatorname{dom} H \subset \mathfrak{H}_{S, A}$. The extension $A_{S}$ of $S$ may or may not depend on $A$. More precisely, we show that the following alternative holds: either $A_{S}=A$ for each selfadjoint extension $A$ of $S$ or $A_{S}$ does not depend on the selfadjoint extension $A$. In this last case $A_{S}$ is called the generalized Friedrichs extension of $S$, cf. also [7]. We then prove that a selfadjoint operator $A$ is semibounded if and only if each one-dimensional symmetric restriction of $A$ has a generalized Friedrichs extension.

We indicate the connection with the function-theoretic approach to the above alternative. If $S$ in (0.1) is determined by $\varphi \in \mathfrak{H}$, then the corresponding nullspace $\operatorname{ker}\left(S^{*}-\ell\right)$ is spanned by

$$
\chi(\ell)=\left(I+(\ell-\mu)(A-\ell)^{-1}\right) \varphi, \quad \ell \in \mathbb{C} \backslash \mathbb{R},
$$

so that $\varphi=\chi(\mu)$. For $a \in \mathbb{R}$ the function

$$
Q(\ell)=a+\bar{\mu}[\varphi, \varphi]+(\ell-\bar{\mu})\left[\left(I+(\ell-\mu)(A-\ell)^{-1}\right) \varphi, \varphi\right]
$$

is a $Q$-function of $A$ and $S$; by definition it is a solution of

$$
\frac{Q(\ell)-\overline{Q(\lambda)}}{\ell-\bar{\lambda}}=[\chi(\ell), \chi(\lambda)]
$$

It belongs to the class $\mathbf{N}$ of Nevanlinna functions, i.e., the class of functions $Q(\ell)$, defined and holomorphic on $\mathbb{C} \backslash \mathbb{R}$, for which $\overline{Q(\ell)}=Q(\bar{\ell})$ and $\operatorname{Im} Q(\ell) / \operatorname{Im} \ell \geq 0$, $\ell \in \mathbb{C} \backslash \mathbb{R}$. We will say that $S$ is of category $\mathbf{N}_{1}$ if there is a selfadjoint extension $A$ whose $Q$-function belongs to the class $\mathbf{N}_{1}$, i.e., if it satisfies $\int_{1}^{\infty} \operatorname{Im} Q(i y) / y d y<\infty$, which is equivalent to $\varphi \in \operatorname{dom}|A|^{\frac{1}{2}}$. Hence, $S$ is of category $\mathbf{N}_{1}$ if and only if for some $Q$-function $Q(\ell)$ the function $\left(Q(\ell)-\tau(\operatorname{Im} Q(\mu))^{2}\right) /(\tau Q(\ell)+1)$ belongs to $\mathbf{N}_{1}$ for some $\tau \in \mathbb{R} \cup\{\infty\}$, see [3]. Moreover, $S$ is of category $\mathbf{N}_{0}$ if there is a selfadjoint extension $A$ whose $Q$-function belongs to the class $\mathbf{N}_{0}$, i.e., if it satisfies $\sup _{y>0} y \operatorname{Im} Q(i y)<\infty$, which is equivalent to $\varphi \in \operatorname{dom} A$. If $Q(\ell) \in \mathbf{N}_{1}$, then $\lim _{y \rightarrow \infty} Q(i y)$ exists and is real, see [5]. The above alternative for a closed symmetric operator $S$ may now be stated as follows: either $S$ is not of category $\mathbf{N}_{1}$, in which case all $Q$-functions belong to $\mathbf{N} \backslash \mathbf{N}_{1}$, or $S$ is of category $\mathbf{N}_{1}$, in which case all but one of its selfadjoint extensions $A$ have a $Q$-function belonging to $\mathbf{N}_{1}$. In this last case, the exceptional selfadjoint extension is the generalized Friedrichs extension; its $Q$-function has the property $\lim _{y \rightarrow \infty} Q(i y)=\infty$. For this function-theoretic approach to generalized Friedrichs extensions we refer to [2], [3].

In Section 1 we prove the above alternative for a symmetric operator in operatortheoretic terms. In Section 2 we prove some auxiliary results concerning a special class of finite measures, which may be of independent interest. The characterization of semibounded selfadjoint operators is given in Section 3 . 


\section{Generalized Friedrichs extensions}

Let $S$ be a closed symmetric operator with defect numbers $(1,1)$ in a Hilbert space $\mathfrak{H}$. In this section we explain the alternative concerning generalized Friedrichs extensions in operator-theoretic terms. Note that $S$ need not be densely defined, so that it may have a proper selfadjoint relation extension $A$ in $\mathfrak{H}$. This means that the multivalued part mul $A=\{g \in \mathfrak{H}:\{0, g\} \in A\}$ is nontrivial; it reduces the relation $A$ and the restriction of $A$ to $\mathfrak{H} \ominus \operatorname{mul} A$ is the operator part, denoted by $A_{s}$, so that $\operatorname{dom} A_{s}=\operatorname{dom} A$ is dense in $\mathfrak{H} \ominus \operatorname{mul} A$.

First assume that $A$ is a selfadjoint operator extension of $S$. Let $\mathfrak{H}_{+1, A}$ be the Hilbert space as defined in (0.4). Clearly, $\operatorname{dom} A$ is dense in the Hilbert space $\mathfrak{H}_{+1, A}$. If $\chi(\mu) \in \mathfrak{H}_{+1, A}$ for some $\mu \in \mathbb{C} \backslash \mathbb{R}$, then the identity

$$
\frac{\chi(\ell)-\chi(\mu)}{\ell-\mu}=(A-\ell)^{-1} \chi(\mu)
$$

shows that $\chi(\ell) \in \mathfrak{H}_{+1, A}$ for all $\ell \in \mathbb{C} \backslash \mathbb{R}$. Since $S$ has defect numbers $(1,1)$ the codimension of $\mathfrak{H}_{S, A}$ in $\mathfrak{H}_{+1, A}$ is at most one. In fact, if $\chi(\ell) \notin \mathfrak{H}_{+1, A}, \ell \in \mathbb{C} \backslash \mathbb{R}$, then

$$
\mathfrak{H}_{S, A}=\mathfrak{H}_{+1, A},
$$

while if $\chi(\ell) \in \mathfrak{H}_{+1, A}, \ell \in \mathbb{C} \backslash \mathbb{R}$, then

$$
\mathfrak{H}_{S, A} \subset \mathfrak{H}_{+1, A}, \quad \mathfrak{H}_{S, A} \neq \mathfrak{H}_{+1, A},
$$

see [2, Theorem 3.1]. It follows from (1.2) and (1.3) that

$$
\operatorname{ker}\left(S^{*}-\ell\right) \cap \mathfrak{H}_{S, A}=\{0\}, \quad \ell \in \mathbb{C} \backslash \mathbb{R} .
$$

To see this, suppose that $\chi(\mu) \in \mathfrak{H}_{S, A}$ for some $\mu \in \mathbb{C} \backslash \mathbb{R}$. This is impossible when $\chi(\ell) \notin \mathfrak{H}_{+1, A}, \ell \in \mathbb{C} \backslash \mathbb{R}$. In the case that $\chi(\ell) \in \mathfrak{H}_{+1, A}, \ell \in \mathbb{C} \backslash \mathbb{R}$, it follows by continuity from (0.2) that

$$
h \in \mathfrak{H}_{S, A} \text { if and only if } h \in \mathfrak{H}_{+1, A} \text { and } \int_{\mathbb{R}}(t-\bar{\mu}) d([E(t) h, \chi(\mu)])=0 .
$$

Now $\chi(\mu) \in \mathfrak{H}_{S, A}$ would imply

$$
\int_{\mathbb{R}}(t-\bar{\mu}) d([E(t) \chi(\mu), \chi(\mu)])=0,
$$

a contradiction.

Now assume that $A$ is a selfadjoint extension of $S$, which is a proper relation. In this case $S$ is nondensely defined and $S^{*}$ is the relation (multivalued operator) defined by

$$
S^{*}=\left\{\{f, g\} \in \mathfrak{H}^{2}:[g, h]=[f, k], \text { for all }\{h, k\} \in S\right\} .
$$

Since $S$ has defect numbers $(1,1)$, mul $S^{*}=\left\{g \in \mathfrak{H}:\{0, g\} \in S^{*}\right\}$ is onedimensional and $A$ is the only proper relation extension of $S$; it is given by

$$
S \dot{+}\left(\{0\} \oplus \operatorname{mul} S^{*}\right),
$$

where $\dot{+}$ stands for the componentwise sum in $\mathfrak{H}^{2}$. Hence mul $A=\operatorname{mul} S^{*}$ and $A_{s}=P S$, where $P$ is the orthogonal projection onto $\mathfrak{H} \ominus \operatorname{mul} S^{*}$. Observe that (1.1) still makes sense with $\chi(\ell) \in \operatorname{ker}\left(S^{*}-\ell\right), \ell \in \mathbb{C} \backslash \mathbb{R}$, and that $\chi(\ell) \notin \overline{\operatorname{dom}} A$, cf. [4]. Since $\mathfrak{H}_{1, A} \subset \mathfrak{H} \ominus \operatorname{mul} A=\overline{\operatorname{dom}} A$, we have $\chi(\ell) \notin \mathfrak{H}_{1, A}$ and (1.4) is still valid. Moreover, $\operatorname{dom} S=\operatorname{dom} A=\operatorname{dom} A_{s}$ shows that (1.2) holds. 
For any selfadjoint extension $A$ of $S$, let $A_{S}$ be the relation associated with $S$ and $A$ by (0.5). Then clearly, $S \subset A_{S} \subset S^{*}$. In order to show that $A_{S}$ is selfadjoint we use von Neumann's formula. There is a one-to-one correspondence between all selfadjoint extensions of $S$ and all $\zeta \in \mathbb{C}$ with $|\zeta|=1$ via

$$
A(\zeta)=S \dot{+} \operatorname{span}\{\chi(\bar{\mu})-\zeta \chi(\mu), \bar{\mu} \chi(\bar{\mu})-\zeta \mu \chi(\mu)\} .
$$

Here $\mu \in \mathbb{C} \backslash \mathbb{R}$ and $\chi(\mu)$ and $\chi(\bar{\mu})$ have the same norm, since $I+(\bar{\mu}-\mu)(A-\bar{\mu})^{-1}$ is a unitary operator. It follows from (1.1) that $\{\chi(\bar{\mu})-\chi(\mu), \bar{\mu} \chi(\bar{\mu})-\mu \chi(\mu)\} \in A$, so that $A$ itself corresponds to $\zeta=1$.

Theorem 1.1. The relation $A_{S}$ is a selfadjoint extension of $S$. In fact, it is the only selfadjoint extension $H$ of $S$ with the property that $\operatorname{dom} H \subset \mathfrak{H}_{S, A}$. The identity

$$
\mathfrak{H}_{S, A}=\mathfrak{H}_{+1, A}
$$

holds if and only if $A_{S}=A$.

Proof. It follows from (1.4) that there is at most one $\zeta \in \mathbb{C}$ for which

$$
\chi(\bar{\mu})-\zeta \chi(\mu) \in \mathfrak{H}_{S, A} .
$$

We claim that there is precisely one $\zeta \in \mathbb{C}$ for which (1.9) holds and we show in that case that $|\zeta|=1$. It then follows from von Neumann's formula that $A_{S}$ is selfadjoint. Moreover, if $H$ is a selfadjoint extension of $S$ such that dom $H \subset \mathfrak{H}_{S, A}$, then $H \subset S^{*}$ implies that $H \subset A_{S}$. Since both $H$ and $A_{S}$ are selfadjoint, we obtain $H=A_{S}$.

To prove our claim, we first consider the case that (1.8) holds. Then, clearly,

$$
A_{S}=\left\{\{f, g\} \in S^{*}: f \in \mathfrak{H}_{+1, A}\right\} .
$$

Since $\chi(\bar{\mu})-\chi(\mu) \in \operatorname{dom} A \subset \mathfrak{H}_{+1, A}=\mathfrak{H}_{S, A}$, it follows that $\zeta=1$ satisfies (1.9).

Next assume that $\mathfrak{H}_{S, A}$ is a proper subspace of $\mathfrak{H}_{+1, A}$, or equivalently that $\chi(\ell) \in$ $\mathfrak{H}_{+1, A}, \ell \in \mathbb{C} \backslash \mathbb{R}$, cf. (1.2) and (1.3). Then the integrals in the following equation are well-defined:

$$
\int_{\mathbb{R}}(t-\mu) d([E(t) \chi(\mu), \chi(\mu)])=\zeta \int_{\mathbb{R}}(t-\bar{\mu}) d([E(t) \chi(\mu), \chi(\mu)]) .
$$

This equation defines a unique $\zeta \in \mathbb{C}$ with $|\zeta|=1$. However, using (1.1) with $\ell=\bar{\mu}$ we see that (1.10) is equivalent to

$$
\int_{\mathbb{R}}(t-\bar{\mu}) d([E(t)(\chi(\bar{\mu})-\zeta \chi(\mu)), \chi(\mu)])=0 .
$$

Due to $(1.5)$ we conclude that $\chi(\bar{\mu})-\zeta \chi(\mu) \in \mathfrak{H}_{S, A}$, so that also in this case (1.9) is satisfied for $|\zeta|=1$.

Finally, note that in the first case when (1.8) holds we have $\zeta=1$ so that $A_{S}=A$, while in the opposite case the identity (1.10) shows that $\zeta \neq 1$ and therefore $A_{S} \neq A$.

Theorem 1.2. Let $S$ be a symmetric operator with defect numbers $(1,1)$. Then the following alternative holds:

(i) The identity (1.8) holds for all selfadjoint extensions $A$ of $S$ and for each selfadjoint extension $A$ of $S$ we have $A_{S}=A$.

(ii) The identity (1.8) holds for precisely one selfadjoint extension of $S$. For each selfadjoint extension $A$ of $S$ the relation $A_{S}$ is independent of $A$. 
Proof. If (1.8) holds for all selfadjoint extensions $A$ of $S$, then according to Theorem 1.1, $A_{S}=A$ for each selfadjoint extension $A$ of $S$.

Now let $A$ be a selfadjoint extension of $S$ for which (1.8) is not valid (so that $A$ and $A_{S}$ are different). Then $\chi(\ell) \in \mathfrak{H}_{+1, A}, \ell \in \mathbb{C} \backslash \mathbb{R}$, and it follows from $[2$, Theorem 4.1], that

$$
\operatorname{dom}|A(\tau)|^{\frac{1}{2}}=\operatorname{dom}|A|^{\frac{1}{2}}
$$

for all $\tau \in \mathbb{R} \cup\{\infty\}, \tau \neq \tau_{0}$, where $\tau_{0} \in \mathbb{R} \cup\{\infty\}$ is the exceptional parameter. By the closed graph theorem, the (topological) spaces $\mathfrak{H}_{+1, A(\tau)}$ and hence, in particular, the spaces $\mathfrak{H}_{S, A(\tau)}$ as point sets are the same for all $\tau \neq \tau_{0}$. Therefore, $A(\tau)_{S}=A_{S}$ for all $\tau \neq \tau_{0}$. Since all $A(\tau), \tau \neq \tau_{0}$, are different from $A_{S}$ and since $A_{S}$ is a selfadjoint extension of $S$, it follows that $A_{S}$ is the exceptional extension corresponding to $\tau_{0}$. For the exceptional selfadjoint extension $A\left(\tau_{0}\right)$ we have $\chi(\ell) \notin \mathfrak{H}_{+1, A\left(\tau_{0}\right)}$, see [3] for the case that $A\left(\tau_{0}\right)$ is an operator and see the above discussion for the case that $A\left(\tau_{0}\right)$ is a proper relation. Hence, equality (1.8) holds and by Theorem 1.1, $A\left(\tau_{0}\right)_{S}=A\left(\tau_{0}\right)=A_{S}$.

Thus, the case where the alternative (i) does not hold gives rise to the alternative (ii).

Due to (1.2) and (1.3) the alternative in Theorem 1.2 can be restated as:

(i) For all selfadjoint extensions of $S$ the corresponding $Q$-functions belong to $\mathbf{N} \backslash \mathbf{N}_{1}$.

(ii) There is precisely one selfadjoint extension (the generalized Friedrichs extension) of $S$ for which the corresponding $Q$-function belongs to $\mathbf{N} \backslash \mathbf{N}_{1}$.

If $S$ is semibounded, the space $\mathfrak{H}_{S, A}$ is the completion of $\operatorname{dom} S$ with respect to the inner product $[(S+a) f, g], f, g \in \operatorname{dom} S$, with $a$ large enough; hence, it is independent of any selfadjoint extension of $S$. In particular, $S$ satisfies the second alternative of Theorem 1.2 and the generalized Friedrichs extension $A_{S}$ coincides with $S_{F}$ in (0.3). If $S$ is nondensely defined (but not necessarily semibounded), it also satisfies the second alternative of Theorem 1.2 and the generalized Friedrichs extension $A_{S}$ is given by (1.6). In this case, all but one of the selfadjoint extensions of $S$ are operators and have a $Q$-function in $\mathbf{N}_{0} \subset \mathbf{N}_{1}$. If, in addition, $S$ is also semibounded, then (1.6) is the Friedrichs extension $S_{F}$ of $S$, cf. [1].

\section{Some AuXiliary RESUlts}

If $d \mu$ is a finite measure and if $h$ is a nonnegative, bounded measurable function on $\mathbb{R}$, then $h d \mu$ is a finite measure and, hence, the function

$$
Q^{h d \mu}(\ell)=\int_{\mathbb{R}}\left(\frac{1}{t-\ell}-\frac{t}{t^{2}+1}\right)\left(t^{2}+1\right) h(t) d \mu(t), \quad \ell \in \mathbb{C} \backslash \mathbb{R},
$$

is well-defined and belongs to $\mathbf{N}$. Moreover, it belongs to $\mathbf{N} \backslash \mathbf{N}_{1}$ if and only if the function $h$ satisfies

$$
\int_{\mathbb{R}}|t| h(t) d \mu(t)=\infty,
$$

which is certainly true when

$$
\int_{0}^{\infty}|t| h(t) d \mu(t)=\infty, \quad \int_{-\infty}^{0}|t| h(t) d \mu(t)=\infty .
$$


In this section we consider finite measures $d \mu$ which satisfy the additional conditions:

$$
\int_{0}^{\infty}|t| d \mu(t)=\infty, \quad \int_{-\infty}^{0}|t| d \mu(t)=\infty
$$

These conditions guarantee the existence of a nonnegative, bounded measurable function $h$ on $\mathbb{R}$ for which the corresponding function $Q^{h d \mu}(\ell)$ in (2.1) has some special limiting properties.

Lemma 2.1. Let $d \mu$ be a finite measure which satisfies (2.4). Then there exists a measurable function $h$ on $\mathbb{R}$ with $0 \leq h(t) \leq 1, t \in \mathbb{R}$, such that (2.3) holds and such that $\lim _{y \rightarrow \infty} \operatorname{Im} Q^{h d \mu}(i y)=0$.

Proof. Define the nonnegative function $\iota(t, y)$ by

$$
\iota(t, y)=y \frac{t^{2}+1}{t^{2}+y^{2}}, \quad y>0, t \in \mathbb{R} .
$$

Clearly, we have, for $y \geq 1$,

$$
\iota(t, y) \leq y, \quad t \in \mathbb{R}
$$

and

$$
\iota(t, y) \leq t^{2}+1, \quad \lim _{y \rightarrow \infty} \iota(t, y)=0, \quad t \in \mathbb{R} .
$$

Note that for all $y \in \mathbb{R}$,

$$
\iota(t, y) \leq|t|, \text { for }|t| \geq 1 .
$$

We construct the function $h$ inductively. Let $u_{1}=1$. Due to (2.4) there exists $v_{1}>u_{1}$ such that

$$
m_{1}^{-}=\int_{\left[-v_{1},-u_{1}\right]}|t| d \mu(t) \geq 1, \quad m_{1}^{+}=\int_{\left[u_{1}, v_{1}\right]}|t| d \mu(t) \geq 1 .
$$

Define the function $h_{1}$ by

$$
h_{1}(t)=\frac{1}{m_{1}^{-}} 1_{\left[-v_{1},-u_{1}\right]}+\frac{1}{m_{1}^{+}} 1_{\left[u_{1}, v_{1}\right]},
$$

so that $h_{1}$ has its support in $\left[-v_{1},-u_{1}\right] \cup\left[u_{1}, v_{1}\right]$ and $\left|h_{1}(t)\right| \leq 1, t \in \mathbb{R}$.

Assume that the function $h_{n-1}$ has been defined, with support contained in

$$
\sum_{k=1}^{n-1}\left(\left[-v_{k},-u_{k}\right] \cup\left[u_{k}, v_{k}\right]\right)
$$

(disjoint union). Since the measure $d \mu$ is finite, we can choose $u_{n}>v_{n-1}$ so large that

$$
\left(\int_{\left(-\infty,-u_{n}\right]}+\int_{\left[u_{n}, \infty\right)}\right) \iota(t, y) d \mu(t) \leq \frac{1}{n}, \quad \text { for all } 1 \leq y \leq u_{n-1},
$$

due to $(2.5)$, and such that

$$
\int_{\left[-v_{n-1}, v_{n-1}\right]} \iota(t, y) d \mu(t) \leq \frac{1}{n}, \quad \text { for all } y \geq u_{n},
$$


due to (2.6) and dominated convergence. Moreover, there exists $v_{n}>u_{n}$ such that

$$
m_{n}^{-}=\int_{\left[-v_{n},-u_{n}\right]}|t| d \mu(t) \geq 1, \quad m_{n}^{+}=\int_{\left[u_{n}, v_{n}\right]}|t| d \mu(t) \geq 1,
$$

due to (2.4). Define

$$
h_{n}=h_{n-1}+\frac{1}{n m_{n}^{-}} 1_{\left[-v_{n},-u_{n}\right]}+\frac{1}{n m_{n}^{+}} 1_{\left[u_{n}, v_{n}\right]},
$$

so that the supports of the summands are disjoint. Clearly $\left(u_{n}\right)$ is a monotonically increasing sequence, with $u_{n} \rightarrow \infty$ as $n \rightarrow \infty$, on account of (2.13). The inductive argument shows that $h(t)=\lim _{n \rightarrow \infty} h_{n}(t)$ is measurable and $0 \leq h(t) \leq 1, t \in \mathbb{R}$. It follows from (2.13) and (2.14) that

$$
\int_{0}^{\infty}|t| h(t) d \mu(t)=\int_{-\infty}^{0}|t| h(t) d \mu(t)=\sum_{k=1}^{\infty} \frac{1}{k}=\infty
$$

so that $(2.3)$ is satisfied.

Finally, we prove the announced limiting behaviour of $Q^{h d \mu}(\ell)$. Let $y \geq 1$ and assume that $y \in\left[u_{n}, u_{n+1}\right]$ (with $n>1$ ) and write

$$
\begin{aligned}
\operatorname{Im} Q^{h d \mu}(i y) & =\int_{\mathbb{R}} \iota(t, y) h(t) d \mu(t) \\
= & \int_{\left[-v_{n-1}, v_{n-1}\right]} \iota(t, y) h(t) d \mu(t) \\
& +\left(\int_{\left[-v_{n+1},-u_{n}\right]}+\int_{\left[u_{n}, v_{n+1}\right]}\right) \iota(t, y) h(t) d \mu(t) \\
& +\left(\int_{\left(-\infty,-u_{n+2}\right]}+\int_{\left[u_{n+2}, \infty\right)}\right) \iota(t, y) h(t) d \mu(t) .
\end{aligned}
$$

Then, due to (2.12) we have

$$
\int_{\left[-v_{n-1}, v_{n-1}\right]} \iota(t, y) h(t) d \mu(t) \leq \int_{\left[-v_{n-1}, v_{n-1}\right]} \iota(t, y) d \mu(t) \leq \frac{1}{n} .
$$

It follows from (2.7), that

$$
\begin{aligned}
& \left(\int_{\left[-v_{n+1},-u_{n}\right]}+\int_{\left[u_{n}, v_{n+1}\right]}\right) \iota(t, y) h(t) d \mu(t) \\
& \quad \leq\left(\int_{\left[-v_{n+1},-u_{n}\right]}+\int_{\left[u_{n}, v_{n+1}\right]}\right)|t| h(t) d \mu(t)=\frac{2}{n}+\frac{2}{n+1},
\end{aligned}
$$

where the last equality follows from (2.13) and (2.14). Finally, due to (2.11) we have

$$
\begin{aligned}
& \left(\int_{\left(-\infty,-u_{n+2}\right]}+\int_{\left[u_{n+2}, \infty\right)}\right) \iota(t, y) h(t) d \mu(t) \\
& \quad \leq\left(\int_{\left(-\infty,-u_{n+2}\right]}+\int_{\left[u_{n+2}, \infty\right)}\right) \iota(t, y) d \mu(t) \leq \frac{1}{n+2} .
\end{aligned}
$$


Hence for the values $y \in\left[u_{n}, u_{n+1}\right]$ we obtain an estimate

$$
\operatorname{Im} Q^{h d \mu}(i y) \leq \frac{3}{n}+\frac{2}{n+1}+\frac{1}{n+2} .
$$

Letting $n \rightarrow \infty$ so that $y \rightarrow \infty$ we conclude that $\lim _{y \rightarrow \infty} \operatorname{Im} Q^{h d \mu}(i y)=0$.

The above lemma gives a construction of a Nevanlinna function whose imaginary part has a specific limiting behaviour. Similarly, we can construct a Nevanlinna function whose real part has a specific limiting behaviour. However, the arguments have to be suitably modified as the reasoning is a little more subtle.

Lemma 2.2. Let $d \mu$ be a finite measure which satisfies (2.4). Then there exists a measurable function $h$ on $\mathbb{R}$ with $0 \leq h(t) \leq 1, t \in \mathbb{R}$, such that (2.3) holds and such that $\lim _{y \rightarrow \infty} \operatorname{Re} Q^{h d \mu}(i y)=0$.

Proof. Define the function $\rho(t, y)$ by

$$
\rho(t, y)=|t| \frac{\left(y^{2}-1\right)}{t^{2}+y^{2}}, \quad y>0, \quad t \in \mathbb{R} .
$$

When $y \geq 1$ this function is nonnegative and, moreover,

$$
\rho(t, y) \leq \frac{y^{2}-1}{2 y}, \quad t \in \mathbb{R}
$$

(where the upper bound itself is monotonically increasing with $y$ ), and

$$
|t|-\rho(t, y)=|t| \frac{t^{2}+1}{t^{2}+y^{2}} \leq|t|, \quad \lim _{y \rightarrow \infty}(|t|-\rho(t, y))=0, \quad t \in \mathbb{R} .
$$

Note that

$$
\rho(t, y) \leq|t|, \quad y \geq 1 .
$$

Now we construct the function $h$. Let $u_{1}=1$. Due to (2.4) there exists $v_{1}>u_{1}$ such that (2.8) holds. Define the function $h_{1}$ by (2.9), so that $h_{1}$ has its support in $\left[-v_{1},-u_{1}\right] \cup\left[u_{1}, v_{1}\right]$ and $\left|h_{1}(t)\right| \leq 1, t \in \mathbb{R}$.

Assume that the function $h_{n-1}$ has been defined, with support contained in the set given by the disjoint union (2.10). Since the measure $d \mu$ is finite, we can choose $u_{n}>v_{n-1}$ so large that

$$
\left(\int_{\left(-\infty,-u_{n}\right]}+\int_{\left[u_{n}, \infty\right)}\right) \rho(t, y) d \mu(t) \leq \frac{1}{n}, \quad \text { for all } 1 \leq y \leq u_{n-1},
$$

due to (2.15), and such that

$$
\int_{\left[-v_{n-1}, v_{n-1}\right]}(|t|-\rho(t, y)) d \mu(t) \leq \frac{1}{n}, \quad \text { for all } y \geq u_{n},
$$

by (2.16) and dominated convergence. Moreover, due to (2.4), there exists $v_{n}>u_{n}$, such that (2.13) holds. Define the function $h_{n}$ by (2.14) (so that the supports of the summands are disjoint). Clearly, $\left(u_{n}\right)$ is a monotonically increasing sequence and $u_{n} \rightarrow \infty$ as $n \rightarrow \infty$, cf. (2.13). Moreover, $h(t)=\lim _{n \rightarrow \infty} h_{n}(t)$ is measurable and $0 \leq h(t) \leq 1, t \in \mathbb{R}$. It follows from (2.13) and (2.14) that for all $n \in \mathbb{N}$ :

$$
\int_{\left[0, v_{n}\right]}|t| h(t) d \mu(t)=\sum_{k=1}^{n} \frac{1}{k}=\int_{\left[-v_{n}, 0\right]}|t| h(t) d \mu(t) .
$$


In particular,

$$
\int_{[0, \infty)}|t| h(t) d \mu(t)=\int_{(-\infty, 0]}|t| h(t) d \mu(t)=\sum_{k=1}^{\infty} \frac{1}{k}=\infty
$$

so that (2.3) is satisfied.

Finally, we prove the announced limiting behaviour of $Q^{h d \mu}(\ell)$. Let $y \geq 1$ and assume that $y \in\left[u_{n}, u_{n+1}\right]$. Then,

$$
\begin{aligned}
& \operatorname{Re} Q^{h d \mu}(i y)=\int_{\mathbb{R}} t \frac{1-y^{2}}{\left(t^{2}+y^{2}\right)} h(t) d \mu(t) \\
& =\int_{\left[0, v_{n-1}\right]}(|t|-\rho(t, y)) h(t) d \mu(t)-\int_{\left[-v_{n-1}, 0\right]}(|t|-\rho(t, y)) h(t) d \mu(t) \\
& \quad-\int_{\left[0, v_{n-1}\right]}|t| h(t) d \mu(t)+\int_{\left[-v_{n-1}, 0\right]}|t| h(t) d \mu(t) \\
& \quad-\int_{\left[u_{n}, \infty\right)} \rho(t, y) h(t) d \mu(t)+\int_{\left(-\infty,-u_{n}\right]} \rho(t, y) h(t) d \mu(t) .
\end{aligned}
$$

Since the integrands of the summands are nonnegative we obtain by using (2.20)

$$
\begin{aligned}
& \left|\operatorname{Re} Q^{h d \mu}(i y)\right| \leq\left(\int_{\left[-v_{n-1}, 0\right]}+\int_{\left[0, v_{n-1}\right]}\right)(|t|-\rho(t, y)) h(t) d \mu(t) \\
& \quad+\left(\int_{\left[-v_{n+1},-u_{n}\right]}+\int_{\left[u_{n}, v_{n+1}\right]}\right) \rho(t, y) h(t) d \mu(t) \\
& \quad+\left(\int_{\left(-\infty,-u_{n+2}\right]}+\int_{\left[u_{n+2}, \infty\right)}\right) \rho(t, y) h(t) d \mu(t) .
\end{aligned}
$$

Due to (2.19),

$$
\begin{gathered}
\left(\int_{\left[-v_{n-1}, 0\right]}+\int_{\left[0, v_{n-1}\right]}\right)(|t|-\rho(t, y)) h(t) d \mu(t) \\
\leq \int_{\left[-v_{n-1}, v_{n-1}\right]}(|t|-\rho(t, y)) d \mu(t) \leq \frac{1}{n} .
\end{gathered}
$$

Due to (2.17),

$$
\begin{aligned}
& \left(\int_{\left[-v_{n+1},-u_{n}\right]}+\int_{\left[u_{n}, v_{n+1}\right]}\right) \rho(t, y) h(t) d \mu(t) \\
& \quad \leq\left(\int_{\left[-v_{n+1},-u_{n}\right]}+\int_{\left[u_{n}, v_{n+1}\right]}\right)|t| h(t) d \mu(t)=\frac{2}{n}+\frac{2}{n+1},
\end{aligned}
$$

where the last equality follows from (2.13) and (2.14). Due to (2.18),

$$
\begin{aligned}
& \left(\int_{\left(-\infty,-u_{n+2}\right]}+\int_{\left[u_{n+2}, \infty\right)}\right) \rho(t, y) h(t) d \mu(t) \\
& \quad \leq\left(\int_{\left(-\infty,-u_{n+2}\right]}+\int_{\left[u_{n+2}, \infty\right)}\right) \rho(t, y) d \mu(t) \leq \frac{1}{n+2} .
\end{aligned}
$$


Hence we obtain

$$
\left|\operatorname{Re} Q^{h d \mu}(i y)\right| \leq \frac{3}{n}+\frac{2}{n+1}+\frac{1}{n+2}, \quad \text { for } y \in\left[u_{n}, u_{n+1}\right] .
$$

Letting $n \rightarrow \infty$ so that $y \rightarrow \infty$ we conclude that $\lim _{y \rightarrow \infty} \operatorname{Re} Q^{h d \mu}(i y)=0$.

Proposition 2.3. Let $d \mu$ be a finite measure which satisfies (2.4). Then there exists a measurable function with $0 \leq h(t) \leq 1, t \in \mathbb{R}$, such that (2.3) holds and such that $\lim _{y \rightarrow \infty} Q^{h d \mu}(i y)=0$.

Proof. Since $d \mu$ satisfies the assumptions of Lemma 2.1, there exists a measurable function $h_{1}$ on $\mathbb{R}$ with $0 \leq h_{1}(t) \leq 1, t \in \mathbb{R}$, such that

$$
\lim _{y \rightarrow \infty} \operatorname{Im} Q^{h_{1} d \mu}(i y)=0, \quad \int_{0}^{\infty}|t| h_{1}(t) d \mu(t)=\infty, \quad \int_{-\infty}^{0}|t| h_{1}(t) d \mu(t)=\infty .
$$

The measure $h_{1} d \mu$ satisfies the assumptions of Lemma 2.2 and hence there exists a measurable function $h_{2}$ on $\mathbb{R}$ with $0 \leq h_{2}(t) \leq 1, t \in \mathbb{R}$, such that

$$
\lim _{y \rightarrow \infty} \operatorname{Re} Q^{h_{2} h_{1} d \mu}(i y)=0, \int_{0}^{\infty}|t| h_{2}(t) h_{1}(t) d \mu(t)=\infty, \int_{-\infty}^{0}|t| h_{2}(t) h_{1}(t) d \mu(t)=\infty .
$$

Since $y \frac{t^{2}+1}{t^{2}+y^{2}}>0$ for $y>0$ and since $h_{1}(t) h_{2}(t) \leq h_{1}(t)$ we see that

$$
\operatorname{Im} Q^{h_{2} h_{1} d \mu}(i y) \leq \operatorname{Im} Q^{h_{1} d \mu}(i y),
$$

for all $y>0$. We conclude that $Q^{h_{2} h_{1} d \mu}(i y) \rightarrow 0$ as $y \rightarrow \infty$.

\section{A CHARACTERIZATION OF SEMIBOUNDEDNESS}

In this section we characterize those selfadjoint operators which are semibounded or bounded in terms of symmetric one-dimensional restrictions.

We first state a result concerning selfadjoint operators which are not semibounded.

Proposition 3.1. Let $A$ be a selfadjoint operator and assume that $A$ is not semibounded. Then there exists an element $\varphi \in \mathfrak{H} \backslash \operatorname{dom}|A|^{\frac{1}{2}}$, such that for the $Q$ function $Q(\ell)$ in (0.6) of $S$ in (0.1) there is a finite limit $\lim _{y \rightarrow \infty} Q(i y) \in \mathbb{R}$.

Proof. Let $\left(E_{t}\right)_{t \in \mathbb{R}}$ be the spectral family of $A$. Since $A$ is not semibounded, the operators $A E_{(-\infty, 0)}$ and $A E_{[0, \infty)}$ are each unbounded, and therefore $\left(-A E_{(-\infty, 0)}\right)^{\frac{1}{2}}$ and $\left(A E_{[0, \infty)}\right)^{\frac{1}{2}}$ are unbounded. Hence there exists an element $\chi \in \mathfrak{H}$ such that

$$
\int_{0}^{\infty}|t| d\left[E_{t} \chi, \chi\right]=\infty, \quad \int_{-\infty}^{0}|t| d\left[E_{t} \chi, \chi\right]=\infty .
$$

The Hilbert space $\mathfrak{K}$, defined by

$$
\mathfrak{K}=\overline{\operatorname{span}}\left\{(A-\mu)(A-\ell)^{-1} \chi: \ell \in \mathbb{C} \backslash \mathbb{R}\right\},
$$

reduces the selfadjoint operator $A$. Since $\chi \in \mathfrak{K}$, it follows that the restriction of $A$ to $\mathfrak{K}$ is not semibounded. Therefore it suffices to prove Proposition 3.1 for the restriction of $A$ to $\mathfrak{K}$. We define the measure $d \sigma$ by

$$
d \sigma(t)=\left(t^{2}+1\right) d\left[E_{t} \chi, \chi\right],
$$


so that

$$
\int_{\mathbb{R}} \frac{d \sigma(t)}{t^{2}+1}<\infty
$$

We associate with $d \sigma$ the Hilbert space $L^{2}(d \sigma)$. Clearly, there is a unitary operator so that the triple

$$
\left(\mathfrak{K},\left.A\right|_{\mathfrak{K}}, \chi\right)
$$

may be identified with the triple

$$
\left(L^{2}(d \sigma), \tilde{A}, \frac{1}{t-i}\right)
$$

where $\tilde{A}$ is the multiplication operator on $L^{2}(d \sigma)$. For any $\varphi \in L^{2}(d \sigma)$ the corresponding $Q$-function with $a=0$ in (0.6) has the form

$$
Q(\ell)=\int_{\mathbb{R}}\left(\frac{1}{t-\ell}-\frac{t}{t^{2}+1}\right)\left(t^{2}+1\right)|\varphi(t)|^{2} d \sigma(t) .
$$

Let $d \mu$ be the finite measure defined by $d \mu(t)=d[E(t) \chi, \chi]$, so that the measure $d \sigma$ is given by $d \sigma(t)=\left(t^{2}+1\right) d \mu(t)$. The condition (3.1) is equivalent to the condition (2.4). According to Proposition 2.3 there is a nonnegative measurable function $h$, such that $0 \leq h(t) \leq 1, t \in \mathbb{R}$, with the indicated properties. Define the function $\tilde{\varphi}(t)$ by

$$
\tilde{\varphi}(t)=\sqrt{\frac{h(t)}{t^{2}+1}}
$$

Then the identity

$$
\int_{\mathbb{R}}|\tilde{\varphi}(t)|^{2} d \sigma(t)=\int_{\mathbb{R}} h(t) d \mu(t)
$$

implies that $\tilde{\varphi} \in L^{2}(d \sigma)$. Let $\varphi \in \mathfrak{H}$ be the element which corresponds to $\tilde{\varphi} \in$ $L^{2}(d \sigma)$. Then for the function $Q(\ell)$ (with $\mu=i$ ) associated with $\varphi(t)$ in $(0.6)$, we find

$$
Q(\ell)=Q^{h d \mu}(\ell)
$$

The identity (2.3) shows

$$
\int_{\mathbb{R}}|t||\tilde{\varphi}(t)|^{2} d \sigma(t)=\int_{\mathbb{R}}|t| h(t) d \mu(t)=\infty .
$$

This implies $\tilde{\varphi} \in L^{2}(d \sigma) \backslash \operatorname{dom}|\tilde{A}|^{\frac{1}{2}}$, so that $\varphi \in \mathfrak{K} \backslash \operatorname{dom}|A|^{\frac{1}{2}}$. Moreover, when $y \rightarrow \infty, Q(i y)$ converges to a real number.

Theorem 3.2. Let $A$ be a selfadjoint operator. Then $A$ is semibounded if and only if each one-dimensional symmetric restriction $S$ of $A$ is of category $\mathbf{N}_{1}$.

Proof. Let $A$ be a semibounded selfadjoint operator. Since each symmetric restriction $S$ of $A$ is also semibounded, it is clear that $S$ is of category $\mathbf{N}_{1}$, see [2].

For the converse we have to show that $A$ is semibounded if each one-dimensional symmetric restriction $S$ of $A$ is of category $\mathbf{N}_{1}$. Assume that $A$ is not semibounded. According to Proposition 3.1 there exists an element $\varphi \in \mathfrak{H} \backslash \operatorname{dom}|A|^{\frac{1}{2}}$ and the $Q$ function $Q(\ell)$ of $A$ and $S$ satisfies $\lim _{y \rightarrow \infty} Q(i y) \in \mathbb{R}$. In particular, this implies that $Q(\ell)$ in (0.6) belongs to $\mathbf{N} \backslash \mathbf{N}_{1}$. If $S$ is of category $\mathbf{N}_{1}$, this means that the 
$Q$-function $Q(\ell)$ is exceptional, so that $Q(i y) \rightarrow \infty$ as $y \rightarrow \infty$. This contradicts $\lim _{y \rightarrow \infty} Q(i y) \in \mathbb{R}$. We conclude that $S$ is not of category $\mathbf{N}_{1}$.

Theorem 3.3. Let $A$ be a selfadjoint operator. Then $A$ is bounded if and only if each one-dimensional symmetric restriction $S$ of $A$ is of category $\mathbf{N}_{0}$.

Proof. Let $A$ be bounded. Then each $\varphi$ in (0.1) belongs to $\operatorname{dom} A$, so that $Q(\ell)$ in (0.6) belongs to $\mathbf{N}_{0}$. Hence, $S$ is of category $\mathbf{N}_{0}$.

Conversely, let $S$ be of category $\mathbf{N}_{0}$. Hence, the $Q$-function $Q(\ell)$ in (0.6) of $S$ and $A$ belongs to $\mathbf{N}_{0}$ (as it clearly is not exceptional, since $A$ is an operator). Equivalently, $\varphi \in \operatorname{dom} A$. Therefore $\mathfrak{H} \subset \operatorname{dom} A$, from which it follows that $A$ is bounded.

\section{REFERENCES}

[1] E.A. Coddington and H.S.V. de Snoo, Positive selfadjoint extensions of positive symmetric subspaces, Math. Z., 159 (1978), 203-214. MR 58:17936

[2] S. Hassi, M. Kaltenbäck, and H.S.V. de Snoo, Triplets of Hilbert spaces and Friedrichs extensions associated with the subclass $\mathbf{N}_{1}$ of Nevanlinna functions, J. Operator Theory, to appear.

[3] S. Hassi, H. Langer, and H.S.V. de Snoo, Selfadjoint extensions for a class of symmetric operators with defect numbers $(1,1), 15$ th OT Conference Proc., (1995), 115-145.

[4] S. Hassi and H.S.V. de Snoo, One-dimensional graph perturbations of selfadjoint relations, Ann. Acad. Sci. Fenn., Series A.I. Math., 22 (1997), 123-164.

[5] I.S. Kac and M.G. Krein, R-functions-analytic functions mapping the upper halfplane into itself, Supplement I to the Russian edition of F.V. Atkinson, Discrete and continuous boundary problems, Mir, Moscow, 1968 (Russian) (English translation: Amer. Math. Soc. Transl., (2) 103 (1974), 1-18). MR 48:6969

[6] T.Kato, Perturbation theory for linear operators, Springer-Verlag, Berlin-Heidelberg-New York, 1966. MR 34:3324

[7] A.G.R. McIntosh, Hermitian bilinear forms which are not semibounded, Bull. Amer. Math. Soc., 76 (1970), 732-737. MR 41:5988

Department of Statistics, University of Helsinki, PL 54, 00014 Helsinki, Finland

E-mail address: hassi@cc.helsinki.fi

Institut für Analysis, Technische Mathematik und Versicherungsmathematik, Technische Universität Wien, Wiedner Hauptstrasse 8-10/114, A-1040 Wien, Österreich

E-mail address: mbaeck@geometrie.tuwien.ac.at

Department of Mathematics, University of Groningen, Postbus 800, 9700 AV GroninGEN, NEDERLAND

E-mail address: desnoo@math.rug.nl 